\title{
Compromiso con la mujer
}

\author{
Magdalena Mut CAMACHO \\ magda.mut@com.uji.es \\ Universitat Jaume I de Castelló
}

Recibido: 31/07/2012

Aceptado: 30/10/2012

\begin{abstract}
Resumen
La sociedad necesita relacionarse con las empresas en un plano de confianza. Pero apostar por este valor como principal peso identificativo y distintivo para presentarse sus los stakeholders debe ser una estrategia que se aborde con firmeza. En este trabajo se trata la combinación entre empresa y mujer puesto que ya se debe superar la igualdad formal y conquistar la igualdad real, un objetivo de más difícil alcance y que requiere de una propuesta identitaria firme por parte de la empresa. El objetivo de este artículo se centra en demostrar que cuando la empresa opta por entender la situación de las mujeres más allá de una mera cuestión formal, comprendiendo que se halla ante una situación que merece una actitud comprometida, absolutamente necesaria y mantenida en el tiempo, se consigue agregar un alto valor positivo.
\end{abstract}

Palabras clave: Mujer, empresa, responsabilidad, compromiso, identidad corporativa

\section{Committed to Women}

\begin{abstract}
The society needs to engage with companies on trust. But bet on this value, needs to be a strategy firmness and neatness. This paper reflects about the combination of business and women. Now is necessary exceed formal equality and conquer real equality. This is more difficult target to reach and requires a firm proposal. The aim of this article is to show that when the company chooses to understand the situation of women beyond a mere matter of form, realizing that is a situation that deserves a committed absolutely necessary and maintained over time, it add a positive value.
\end{abstract}

Keywords: Woman, enterprise, responsibility, commitment, corporate identity

\section{Referencia normalizada}

MUT CAMACHO, Magdalena (2012): “Compromiso con la mujer". Estudios sobre el mensaje periodístico. Vol. 18, núm. especial noviembre, págs.: 629-637. Madrid, Servicio de Publicaciones de la Universidad Complutense.

Sumario: 1. Introducción. 2. Metodología y objetivos. 3. Identidad corporativa y mujer. 4. Empresa, beneficios y mujer. 5. Estudio del caso Deloitte: ejemplo de compromiso real. 6. Conclusiones para un debate. 7. Referencias bibliográficas

\section{Introducción}

Hoy en día, la confianza se ha convertido en un valor esencial en todas las organizaciones, pero apostar por este valor como principal peso identificativo y distintivo para presentarse ante los stakeholders es una estrategia que abordan con gran firmeza algunas empresas.

Esta decidida postura se fundamenta en que la actitud y la forma de ser de la empresa, desempeña un papel de gran importancia en el plano social. Es más, algunas teorías defienden a la empresa como uno de los actores más potentes en la producción de la cultura de la sociedad contemporánea. En esta línea y centrándonos en la igualdad de oportunidades, el objetivo de este artículo se centra en demostrar que cuando la empresa opta por favorecer la situación de las mujeres más allá de una mera cuestión formal, conquistando la igualdad real y comprendiendo que se haya ante una situación 
que merece una actitud diferente, absolutamente necesaria y mantenida en el tiempo, se consigue agregar un valor positivo al conjunto, a la empresa y a la sociedad.

\section{Metodología y objetivos}

La metodología que utiliza combina el análisis de la teoría sobre la responsabilidad social y los estudios vinculados a la igualdad, junto con el contraste de conclusiones a través del estudio de un caso concreto, como es el tratamiento de la situación laboral de las mujeres en la empresa Deloitte.

El objetivo del presente artículo es extraer conclusiones tras descubrir cómo la responsabilidad de las organizaciones en cuestiones de sexo, ofrece beneficios evidentes en una doble perspectiva, la reputación interna y la externa. En el plano internos, para la organización significa el aprovechamiento de todo el talento que de otra forma sería desaprovechado; y desde la perspectiva externa incide en el incremento de la reputación corporativa, fundamentada en esa firme actitud ética que exhibe la organización, además, una empresa socialmente responsable está legitimada por la sociedad para operar en ella y admirarla.

\section{Identidad corporativa y mujer}

La identidad corporativa de una organización se concreta en su estilo de ser, en su forma de comportarse, esta actitud emana de lo que Schein (1988) llama el nivel más profundo de presunciones básicas y creencias. La "psique corporativa" es la explicación que Villafañe propone, definiéndola como el lugar que alberga todo tipo de cuestiones (comportamientos, actitudes, valores, creencias, costumbres, presunciones, etc.) observables, es la "conciencia corporativa". Sin embargo, también señala que existe otro tipo de manifestaciones que no son tan visibles, pues están alojadas en el inconsciente corporativo. Ambas configuran la "cultura corporativa" de una organización (Villafañe, 1999).

En una organización todo lo estratégico, todo lo importante, emana de su identidad corporativa. Por tanto, las cuestiones relacionadas con la responsabilidad emanan de lo más profundo de la empresa, del lugar donde residen las convicciones, más allá del tiempo y de las personas que hay y habrán. Es en este lugar donde se ha de escribir cual es la actitud de la empresa ante la igualdad de oportunidades para hombres y mujeres en su gestión funcional. Porque si no es así, la empresa no actuará en consecuencia de una forma natural y sincera, sin esta disposición anclada en su identidad no será una firme convicción el querer ofrecer la igualdad de oportunidades al entender con ecuanimidad la relación de las mujeres, su carrera profesional y su vida. Esto es muy importante porque es en este anclaje, o en esta falta de anclaje, de donde surgen las dudas de la sociedad sobre el verdadero interés de las empresas en ser responsables.

Existen múltiples estudios que demuestran que las nuevas orientaciones empujan a las organizaciones al cuidado de su capital humano como factor estratégico de desarrollo (Guthridge, 2008). Entre las tendencias que más valor reputacional van otorgando con el tiempo, encontramos el talento como emergente (Villafañe, 2007). Los directores generales de las empresas asumen que la carencia de talento impacta en la 
productividad pero también es un motivo de pérdida de oportunidades (PWC, 2012: web). De forma que el objetivo es alinear la estrategia y el compromiso, por lo tanto, el reto para las organizaciones está en entender cual es la capacidad e idiosincrasia que tiene el compromiso de los empleados para generar beneficios en el negocio.

Un estudio llevado a cabo por Corporate Executive Board encontró que los empleados que estaban más comprometidos con sus organizaciones se esforzaban un $57 \%$ más y existía un $87 \%$ de menos probabilidades de renunciar a su trabajo.

\section{Empresa, beneficios y mujer}

Si bien no existen estudios concluyentes que asocien causa y consecuencia entre responsabilidad social corporativa y beneficios (de ahí que hayan distintas posturas a favor y en contra), sí existen indicios que nos ayudan a vislumbrar una postura estable y consecuente. El índice FTSE4Good descubre que las empresas con estándares de responsabilidad corporativa tienen beneficios superiores a las que no y también hay diferentes estudios apuntan a este resultado (Margolis et al., 2003).

Lo cierto es que los consumidores prefieren marcas de empresas responsables, los ciudadanos prefieren trabajar en empresas responsables y éstas retienen mejor el talento, las empresas con códigos de conducta para proveedores garantizan estándares tanto en sus producciones como en la transmisión de valores hacia la cadena implicada en la producción, etc. En algunos casos esta vigilancia sirve para mejorar condiciones de trabajadores y comunidades donde se ubica la empresa proveedora, como se ha visto recientemente con Apple (RTVE.es, 2012: web).

Tom Peters (2006) tachaba de "patético" el número de mujeres que participaban en las altas direcciones, cifrándolo en EEUU en un 4\%, en Gran Bretaña en un 3\% y en Japón en un 1\%. En España sigue existiendo un escaso número de mujeres en los comités ejecutivos (únicamente el $6 \%$ y muchas de ellas ocupan este cargo por herencia del negocio familiar) y en los consejos de administración de las empresas, en este último caso, en nuestro país el porcentaje es del 8\%, en Europa es del 12\% (lavanguardia.es, 2011: web), cifras que siguen siendo muy bajas; por eso desde la Comisión Europea se exigen reiteradamente que se incorporen más mujeres en los consejos de administración.

La Ley de Igualdad ha favorecido la presencia de mujeres en los consejos de administración y organismos de decisión de las empresas españolas si bien aún a un ritmo lento. A pesar de que las mujeres representan un $44 \%$ de la población activa y un $50,7 \%$ de la total, y de que su nivel de formación a la hora de integrarse en el mercado laboral sea mayor que el de los hombres, su presencia en los órganos de administración empresarial no ve reflejada esta realidad (INE, 2010). La Unión Europea se ha fijado el "Objetivo 2015" para alcanzar la cifra del 30\% de mujeres en los consejos de administración.

Esta cuestión es generalizada en muchos países, el informe McKinsey "Women Matter" 2010 ve mejores resultados en las empresas con diversidad de género. Muestra que las empresas que registran un mayor porcentaje de mujeres en los comités ejecutivos tienen un $41 \%$ más de rentabilidad y un $56 \%$ más de margen de beneficios que las empresas que no tienen a mujeres en sus cúpulas directivas. 
Otro dato a favor lo desvela un informe de Goldman Sach, donde se señala que si la Unión Europea suavizara la diferencia laboral y salarial entre hombres y mujeres, el PIB europeo aumentaría un 13\% (rcysostenibilidad.org, 2011: web).

Por tanto, los estudios demuestran que las compañías que mantienen programas de diversidad son más productivas y se sienten más motivadas. Además de que estos programas benefician la reputación de la empresa en la sociedad, y mantienen y retienen el talento por lo tanto.

\section{Estudio del caso Deloitte: ejemplo de compromiso real}

La empresa Deloitte es una consultora de servicios profesionales implantada en todo el mundo. Define su misión como "ser el estándar de excelencia" y su visión es "firma líder del mercado español, reconocida por ser el asesor de referencia de las principales entidades a través de una oferta de servicios de alto valor, y por su capacidad de desarrollo del mejor equipo humano" (Deloitte, 2012: web).

En 2005 Deloitte se convirtió en la primera firma en España que publicaba una Memoria de Responsabilidad Corporativa, y en ella recogía toda la información relacionada con la gestión de su capital intelectual. Desde entonces, cada año, ha continuado reforzando su compromiso con la transparencia y la sostenibilidad a través del diálogo con sus grupos de interés, evidenciado en sus memorias. Por otra parte, se acogen al modelo del GRI y obtienen la máxima calificación (A+).

Deloitte considera que el diálogo con sus grupos de interés es necesario para una mejora continua de su relación con los mismos, de forma que la firma renueva cada año sus compromisos con la sociedad y asume nuevos retos que incorpora a su gestión, esta es su forma de innovar en la gestión.

En este sentido, el título que lleva su última memoria publicada, "El valor del compromiso", es una frase virtuosa que muestra cómo las cuestiones relativas a la identidad de la empresa son posturas y actitudes que van más allá de meras acciones, son auténticas creencias compartidas y por tanto, defendidas y predicadas.

En sus señas identitarias tiene asumida la importancia del esfuerzo y el compromiso de todos los profesionales de la firma, por ello el capital humano forma parte importante de su estrategia. La empresa descubre que en el desarrollo del tejido empresarial español y la competitividad de las compañías, juega un papel principal el compromiso con el empleado, por tanto merece una visión muy comprometida la gestión de la cultura corporativa. Con lo cual, la firma ofrece oportunidades de desarrollo de capacidades a su gente, proporcionado formación para el desarrollo personal y profesional, contribuyendo a su especialización para sentirse parte importante en el devenir de la compañía y fomentando el trabajo en equipo con procesos eficientes. El resultado de todo ello es la aportación, por derivación, de valor añadido a sus clientes, beneficios para la empresa y confianza y bienestar social.

En este año pasado, el porcentaje de puestos de gerencia ocupados por mujeres en Deloitte ha sido de un $28 \%$, bastante más elevado que la media española, y más del doble de la europea.

En Deloitte la igualdad de oportunidades ha sido desde hace tiempo uno de sus rasgos más característicos. Todo empezó hace 20 años cuando la empresa fue cons- 
ciente de una serie de cuestiones que afectaban a sus procesos, a sus resultados y a su identidad, sobre las que se debía meditar en profundidad para extraer una postura comprometida. Por un lado, la evidente comprensión de que el 50\% del talento del mundo está en el sexo femenino y, por otro, la percepción de que la riqueza creativa está en los abundantes y diversos puntos de vista. Por otra parte, la empresa en su experiencia acumulada en su historia corporativa, descubría que las mujeres abandonaban voluntariamente sus carreras profesionales por motivos personales varios como la priorización de la maternidad, la diferente lógica sobre que es la competencia entre profesionales en la empresa, la no asunción del trabajo como disponibilidad anytimeanywhere como sinónimo de alta responsabilidad profesional y mejor predisposición ante su empleador, entre otros variados motivos vinculados a su condición de mujer.

En ese momento de clarividencia de la situación, reflexión y actuación, la empresa comenzó su propuesta de cambio recogiendo todos los problemas entorno a la situación de la mujer y se llevaron a debate interno. Por otra parte, el entendimiento y aceptación de que hay cuestiones en la identidad corporativa que son dinámicas, llevó consecuentemente a repensar su cultura corporativa y adaptarla a la realidad más allá de simples gestos, sino como una cuestión principal a la que atender de forma profunda y comprometida.

Se puso en marcha el plan Women's Initiative con el objeto de conseguir retener y promover a la mujer en la empresa. Todo ello supuso una revolución cultural que zarandeo los cimientos de la empresa. Se apostó por la flexibilidad de horarios, por apoyar una mayor representación y liderazgo de la mujer, se buscó siempre el consenso en las decisiones e incluso se adoptó la actitud de bajar hasta el último peldaño de personalización del problema de la empleada si era necesario.

Deloitte se ha convertido una de las primeras empresas más admiradas para ir a trabajar, saliendo en los rankings Great Place to Work que edita la revista Fortune anualmente, y en Great Place to Work for Mothers.

Como evidente ejemplo de su compromiso identitario con las cuestiones que conciernen a las mujeres, Deloitte ha sabido impregnar de esta seña identitaria también al servicio que ofrece a sus clientes, consiguiendo un doble resultado, valor añadido en su servicio y mejora social en el entorno. Deloitte está implantada en todo el mundo y la transferencia de conocimiento y procesos de gestión los lleva hasta el último rincón del planeta, donde han diferentes situaciones sociales para las mujeres. Para ello posee tres herramientas básicas: Global Retention and Advancement of Women Council (GROW), the Global Diversity and Inclusion Community of Practice (CoP) y la celebración del International Women's Day (IWD) cada año. Con estas iniciativas aúna los esfuerzos y los orienta hacia objetivos concretos para el apoyo e impulso de las mujeres, fomentando herramientas, recursos, programas, etc. en todos los lugares donde opera.

El International Womes's Day es un evento que se celebra todos los años el 8 de marzo, en él se celebran los logros económicos, políticos y sociales de las mujeres del pasado, presente y futuro. Deloitte reúne a un grupo de líderes empresariales de todo el mundo para discutir con información y perspectivas novedosas asuntos cómo el fomento del desarrollo de las mujeres como trabajadoras, como líderes o como consumidoras, y como ello puede tener beneficios significativos. 
El know how de Deloitte le ha llevado a participar en el desarrollo del modelo de gestión de los principios del Global Compact. Trabajaron con el fin de conseguir un modelo para guiar a las empresas de todos los tamaños a través del proceso hacia la sostenibilidad corporativa, mediante la ejecución de los diez principios del Pacto en sus operaciones y cultura organizacional. Elaboraron una guía para retener e impulsar a las mujeres en el lugar de trabajo, en el mercado y en la comunidad. Desde este documento se hace hincapié en la necesidad de promover la igualdad de género y empoderamiento de la mujer en las empresas.

En Deloitte su posición comprometida ante la situación de la mujer forma parte de su identidad, y de ahí, de su estrategia de negocio. Ha comprendido que la diversidad es fuerza y ha sabido explotarla e incrementarla en beneficio suyo y de sus clientes (Deloitte, 2012: web).

\section{Conclusiones para un debate}

Los estereotipos de género impulsan a las personas a adquirir determinados comportamientos o roles de género, que han comportado la segregación ocupacional entre hombres y mujeres en función de su sexo (Bernard, 2007: 215). De las empresas depende el bienestar y el desarrollo de las personas, ya que buena parte del tiempo de su vida lo pasan en su puesto de trabajo, "por tanto, si pretendemos aumentar no sólo el valor económico, sino también los niveles de bienestar de las personas en todos los aspectos de su vida, es preciso reconocer que su bienestar en el trabajo depende en parte de la calidad del management, que coordina sus actividades y que al final es responsable de su desarrollo, tanto profesional como personal" (Andreu y Rosanas, 2011: 6)

El principio de igualdad es un derecho fundamental de las personas, y en este sentido, ningún ser humano puede ser discriminado por razón de sexo. Aunque esto ya sea una certeza, las empresas han de entender que actuar con igualdad es más que una postura formal, es un compromiso real. La empresa de hoy ha de mantener una actitud con valores firmes si se desea que éstos sean creíbles. Estos valores han de estar sólidamente arraigados en su identidad corporativa, sólo así serán palanca de cambio y proyección, además de escudo de protección (Mut y Bernad, 2012: 609).

Pese al tratamiento legal y al reconocimiento de la igualdad por parte de las empresas, impidiendo de este modo situaciones de discriminación directa contra las mujeres, no es menos cierto, que se parte de una situación de desventaja. El género es una construcción cultural que se ha plasmado históricamente en forma de dominación masculina y sujeción femenina. Esta jerarquización sexual se ha materializado en sistemas sociales y políticos patriarcales (Cobo, 1995: 56). Apoyándose en esta definición, Bernad y López Lita (2007: 220) destacan que esta construcción cultural se ha ido forjando a lo largo de la historia, en torno a una serie de componentes de género: el rol, o componente sociológico; la identidad sexuada, o componente psicológico, y el estatus o componente político, que han venido reproduciendo elementos funcionales como los estereotipos de género, que determinan la reproducción social de la desigualdad entre ambos géneros.

Hasta no hace mucho tiempo, las compañías establecían como máxima meta de su existencia el lograr los mejores resultados en el corto plazo, así que sólo miraban sus 
resultados financieros, si disponían de las más capaces cadenas de producción y si eran propietarias de las instalaciones más eficientes. Ahora ha variado el rumbo, en este nuevo siglo, los valores y la cultura de la sociedad cambian de día en día. A consecuencia de esto, hoy sabemos que la reputación que tenga una compañía supone más del 70\% de la decisión de compra de los consumidores finales (Atienza, 2010).

Por tanto, las empresas han de estar atentas a estos cambios en los valores sociales para poder integrarlos en su evolutiva y dinámica identidad corporativa (Schein, 1988).

En otro orden de cosas, pero con el mismo resultado, en la actualidad la RSC se ha convertido en una de las variables que más diferenciación aporta a las organizaciones en las métricas sobre reputación corporativa (Villafañe, 2007). Con frecuencia más organizaciones, ante los retos crecientes a los que se enfrenta, son capaces de diseñar proyectos y actuaciones de responsabilidad corporativa eficientes y que aportan valor a la mejora de su competitividad, a la minimización de su huella ecológica, al apoyo a la sociedad, especialmente a los sectores más desfavorecidos, y a la consolidación y fortalecimiento de la gestión ética y responsable.

Por tanto, el apostar por una actitud comprometida con las necesidades de las mujeres en el trabajo, eliminando cualquier rastro de género, significa el tener una actitud responsable que sirve a la diferenciación y a la estima de los públicos de interés de la empresa, a la admiración en su fin último y máximo.

Se descubre que abrir las puertas al talento es una forma de gestionar el mañana. Las mujeres suponen la mitad del talento de la sociedad y las empresas han de conseguir retenerlo. En relación con la rentabilidad, se ha demostrado que las empresas que tienen más mujeres en puestos de decisión tienen mayores rentabilidades (Wittenberg, 2008).

Desde la eficiencia y la excelencia, todo aquello referente a la igualdad de oportunidades en lo referente a la idiosincrasia de la mujer a la hora de desempeñar un trabajo va más allá de únicamente adoptar en la empresa una serie de cuestiones estándares sobre funcionamiento de recursos humanos. Descubrir qué barreras de participación y de rendimiento tienen las mujeres es indagar más y mejor en la cuestión diferencial desde una óptica de integración y comprensión, que ha de estar bien arraigada en la identidad corporativa de la organización.

\section{Referencias bibliográficas}

ANDREU, Rafael y ROSANAS, Josep Maria (2011): Manifiesto para un management mejor. Una visión racional y humanista. Madrid, IESE Business School.

ATIENZA, Luis. (2010): "Prólogo", en Catálogo de buenas prácticas en Responsabilidad Corporativa. Madrid, Club de la Excelencia.

BERNAD, Estela y LÓPEZ LITA, Rafael (2007): "Publicidad, medios de comunicación y segregación ocupacional de la mujer: perpetuación y superación de los estereotipos de género y sus consecuencias en el mercado de la mano de obra". Revista del Ministerio de Trabajo y Asuntos Sociales. Madrid, pp. 213-227. 
DELOITTE (2012): "Diversity and Inclusion", en http://www.deloitte.com/view /en_GX/global/about/diversity-inclusion/index.htm [fecha de consulta: 19 de febrero de 2012]

EL ECONOMISTA.ES. (15 de 01 de 2007): "RSC-Se entregan este mes los Premios 'Ojo Público' de Davos en el Foro Económico Mundial a las empresas menos ejemplares", en http://www.eleconomista.es/mercados-cotizaciones/noticias/131470 /01/07/RSC-Se-entregan-este-mes-los-Premios-Ojo-Publico-de-Davos-en-el-ForoEconomico-Mundial-a-las-empresas-menos-ejemplares.html

ELMUNDO.ES (22 de junio de 2004): "Wal Mart, ante la mayor demanda colectiva por discriminación", en: http://www.elmundo.es/mundodinero/2004/06/22 /Noti20040622192437.html

GUTHRIDGE, Matthew (2008): "Making talent a strategic priority", en http://www.mckinseyquarterly.com/Making_talent_a_strategic_priority_2092

FORÉTICA (2011). Igualdad y RSE. Guía para PYMES: Forética, Madrid.

INSTITUTO NACIONAL DE ESTADÍSTICA (INE) (2010): Mujeres y Hombres en España 2010. Madrid.

LAVANGUARDIA.COM (01 de 03 de 2011). "España por debajo de la media en mujeres en consejos de administración", en http://www.lavanguardia.com/economia/20110301/54120874403/espana-por-debajo-de-la-media-en-mujeres-en-consej os-de-administracion.

MARGOLIS, Joshua y WALSH. James (2003): "Misery loves Companies: Rethinking Social Initiatives by Business". Administrative Science Quaterly, Volumen vol. 48, pp. 268-305.

MUT, Magdalena y BERNAD, Estela (2012): "El mensaje comprometido es otra publicidad". Revista de Comunicación Vivat Academia, XIV(Especial). Madrid, pp. 598-611.

PETERS, Tom. (2006): Re-imagine. Business excellence in disruptive age. Madrid, Pearsons Educación.

RCYSOSTENIBILIDAD.ORG (08 de 03 de 2011): "Blog RC y sostenibilidad", en http://www.rcysostenibilidad.telefonica.com/blogs/2011/03/08/dia-internacionalde-la-mujer-la-igualdad-es-un-buen-negocio-english-version-follows/

RCYSOSTENIBILIDAD.ORG (03 de 08 de 2011): “Día internacional de la mujer", en http://www.rcysostenibilidad.telefonica.com/blogs/2011/03/08/dia-internacionalde-la-mujer-la-igualdad-es-un-buen-negocio-english-version-follows/

RTVE.ES (16 de 01 de 2012): “Apple desvela por primera vez su lista de proveedores", en http://www.rtve.es/noticias/20120116/apple-desvela-primera-vez-listaproovedores-para-frenar-criticas/489919.shtml

SCHEIN, Edgar. (1988): La cultura empresarial y liderazgo. Una visión dinámica. Barcelona, Plaza y Janés. 
UNIÓN EUROPEA (2001): Libro verde. Fomentar un marco europeo para la responsabilidad social de las empresas. Bruselas, Unión Europea.

VILLAFAÑE, Justo. (2007): "La gestión intangible", en VILLAFAÑE. Justo., Informe anual 2007. La comunicación empresarial y la gestión de intangibles. Madrid, Pirámide.

WITTENBERG, Avivah (2008): Why women mean business. San Francisco CA, Jossey-Bass.

WORLD BUSINESS COUNCIL FOR SUSTAINABLE DEVELOPEMENT (2010): Visión 2050. Un nueva agenda para los negocios. Bruselas, WBCSD. 